Research Paper

\title{
Higher Plasma Fibrinogen Levels are Associated with Malignant Phenotype and Worse Survival in Patients with Glioblastomas
}

\author{
Peng-Fei Wang1\#, Zhe Meng1\#, Hong-Wang Song1, Kun Yao², Ze-Jun Duan², Shou-Wei Li1 ${ }^{\bowtie}$, Chang-Xiang \\ $\mathrm{Yan}^{1 凶}$ \\ 1. Department of Neurosurgery, Sanbo Brain Hospital, Capital Medical University, China; \\ 2. Department of Pathology, Sanbo Brain Hospital, Capital Medical University, China. \\ \#These authors contributed equally to this work. \\ $\triangle$ Corresponding authors: Shou-Wei Li, Email: 15011339604@163.com; Phone: 0086-010-62856706; Fax: 0086-010-62856716. Address: Building 1, Ward 6, Xiang \\ Shan Yi Ke Song Road 50, Haidian, Beijing, China; Chang-Xiang Yan, Email: yancx65828@sina.com; Phone: 0086-010-62856706; Fax: 0086-010-62856716. Address: \\ Building 1, Ward 6, Xiang Shan Yi Ke Song Road 50, Haidian, Beijing, China \\ (c) Ivyspring International Publisher. This is an open access article distributed under the terms of the Creative Commons Attribution (CC BY-NC) license \\ (https://creativecommons.org/licenses/by-nc/4.0/). See http://ivyspring.com/terms for full terms and conditions.
}

Received: 2018.01.03; Accepted: 2018.03.05; Published: 2018.04.30

\begin{abstract}
Preoperative plasma fibrinogen levels were associated with poor clinical outcomes in malignancies. There were few data about the prognostic value of plasma fibrinogen in glioblastomas (GBMs). The objective of our study was to investigate the association between fibrinogen and patients' clinicopathological factors and overall survival (OS). From 2008 to 2016, 315 patients with GBMs who had a surgical treatment at our institute, were retrospectively involved in this study. IDH (isocitrate dehydrogenase) mutations and ATRX (alpha thalassemia/mental retardation syndrome X-linked) loss were detected with IHC (Immunohistochemistry). The preoperative plasma fibrinogen levels ranged from 1.00 to $5.22 \mathrm{~g} / \mathrm{L}$, with a mean of were $2.57 \mathrm{~g} / \mathrm{L}$. There were increased levels of plasma fibrinogen in patients aged $\geq 65$ years, secondary GBMs, IDH mutation $(p=$ $0.033)$ and ATRX loss $(p=0.040)$. Moreover, the plasma fibrinogen level was the highest in the subtype of IDH- 1 RI32H wildtype - ATRX expression, which showed a shorter OS compared to the group of IDH- $1 \mathrm{R} 132 \mathrm{H}$

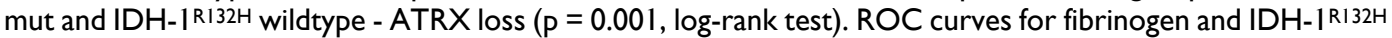
wildtype - ATRX expression was also plotted, and indicated a potential diagnostic value of fibrinogen in molecular pathology. Univariate analysis found that younger age, higher KPS (Karnofsky Performance Score), gross total resection, complete chemoradiotherapy, IDH-1RI32H mutations and lower levels of fibrinogen were associated with favorable outcomes. Multivariate analysis proved that chemoradiotherapy, IDH-1RI32H and fibrinogen were independent prognostic factors. In conclusion, plasma fibrinogen could predict clinical outcome and molecular subtype in GBMs.
\end{abstract}

Key words: Glioblastomas; Fibrinogen; IDH mutations; ATRX loss; Prognosis.

\section{Introduction}

Gliomas were the most common brain cancers [1]. Glioblastomas (GBMs) constituted the majority of gliomas, which were characterized by high rates of recurrence and poor prognoses [2]. Current treatment strategies for GBMs mainly included surgery, radiotherapy and chemotherapy; but there was no progress of overall survival in glioma patients [3]. Molecular pathology of gliomas had made great progress in predicting the prognosis and guiding treatment strategy $[2,4]$. However, a major limitation in practice use was that it could be only evaluated after surgery. In comparison, circulating biomarkers were easy-obtained and effective, and showed prognostic value for cancer patients, such as neutrophil-to-lymphocyte ratio (NLR) and fibrinogen $[5,6]$.

There were numerous studies suggested that high plasma fibrinogen levels were associated with worse clinical outcome in a variety of solid tumors [6]. The prognostic value of plasma fibrinogen was also 
proven in human gliomas [7, 8]. Nevertheless, the reliability and degree of this prognostic marker needed to be re-evaluated, due to limited cases and variation in study designs. Consequently, we retrospectively analyzed the clinical data of 315 glioblastomas patients. The association between plasma fibrinogen and tumor prognosis, IDH (isocitrate dehydrogenase) mutations and ATRX (alpha thalassemia/mental retardation syndrome $\mathrm{X}$-linked) loss were also investigated here.

\section{Methods}

\section{Study population}

From 2008 to 2016, 315 patients with glioblastoma were retrospectively involved in this study. All the patients had a surgical removal at Sanbo Brain Hospital, and the diagnosis of GBM was based on pathology [2]. The adjuvant chemoradiotherapy was administrated according to Stupp's protocols [9]. The complete adjuvant therapy was defined as patients who finished full concurrent chemoradiotherapy plus 2 more cycles' consolidation temozolomide; the counterparties were the group of incomplete adjuvant therapy [10]. Overall survival (OS) was defined as the period from operation to death or censored. The deadline of our follow-up was Nov, 2017. All the procedures in this study were approved by the ethics committee of Sanbo Brain Hospital. The written informed consent was obtained from all patients.

\section{Evaluation of IDH-1 ${ }^{\mathrm{R} 132 \mathrm{H}}$ mutations and ATRX loss}

The Immunohistochemistry (IHC) was performed to evaluate the IDH-1 ${ }^{\mathrm{R} 132 \mathrm{H}}$ mutations and ATRX loss in GBMs, according to our previous report [11]. Briefly, $4-\mu \mathrm{m}$-thick sections were prepared from formalin-fixed, paraffin-embedded tumor tissue. Primary antibodies against IDH-1 ${ }^{\mathrm{R} 132 \mathrm{H}}$ (Dianova 1:100) and ATRX (Sigma 1:1000) were applied overnight at $4^{\circ} \mathrm{C}$. Then, the sections were washed with PBS, and incubated with poly-HRP Anti-Mouse/Rabbit IgG Detection System (PV-9000 ZSGB-BIO, China) for 30 $\min$ at $37^{\circ} \mathrm{C}$. The pathological outcome was evaluated independently by two experienced pathologists. The cutoff value of IDH-1 $1^{\mathrm{R} 132 \mathrm{H}}$ and ATRX was $10 \%$.

\section{Statistical analysis}

SPSS 22.0 and GraphPad Prism 5 were the main tools for analyzing data and drawing figures. The student test was used for appropriate data analysis. Overall survival was analyzed with Kaplan-Meier method, and further confirmed by log-rank test. The optimal cutoff for plasma fibrinogen in predicting OS in GBM was evaluated by cutoff finder [12]. Cox proportional hazards models were adopted for calculating the hazard ratios (HRs) of death in GBMs with regard to univariable or multivariable analysis. $\mathrm{P}<0.05$ was considered statistically significant.

\section{Results}

\section{Baseline characteristics}

A total of 315 GBM patients, which consisted of 257 primary GBMs and 58 secondary GBMs were involved in our study. The median age was 51.0 years and $39.4 \%(124 / 315)$ patients were females. In the KPS (Karnofsky Performance Score), 64.4\% (203/315) patients scored $70-100$, and $35.6 \%$ (112/315) patients scored less than 70 . The gross total resection (GTR) and non-GTR was achieved in $64.3 \%(196 / 305)$ and $35.7 \%(109 / 305)$ patients respectively. While 58.2\% $(173 / 297)$ had a complete adjuvant therapy, $41.8 \%$ $(124 / 297)$ patients failed to do this. There were 77 patients carrying on IDH-1 ${ }^{\mathrm{R} 132 \mathrm{H}}$ mutations, and 238 cases without these mutations. ATRX loss was found in $19.9 \%(60 / 301)$ patients and expression was detected in $80.07 \%(241 / 301)$ patients.

\section{Association of plasma fibrinogen and clinicopathological factors}

The mean preoperative plasma fibrinogen levels were $2.57 \mathrm{~g} / \mathrm{L}$ with a range of 1.00-5.22 g/L. There was no difference of plasma fibrinogen levels in gender, preoperative KPS, tumor size, resection extent, or a complete chemoradiotherapy (Table 1). However, the decreased plasma fibrinogen was significantly associated younger age $(p=0.042)$ and sGBM ( $p=0.002)$. Moreover, we found a positive correlation of age with fibrinogen $(p<0.001$, Fig. Supplement 1). It was well acknowledged that IDH mutation and ATRX loss were the most important genetic alternations in GBMs [13]. We found that OS was significantly improved in patients with IDH-1 ${ }^{\text {R132H }}$ mutation (19.93 months, 95\% CI 13.62-26.24 vs 12.30 months, $95 \%$ CI 10.86-13.74; $\mathrm{p}<$ 0.001; Fig.1A), but failed in ATRX loss (16.23 months 95\% CI 12.16-20.30 vs 12.80 months, 95\% CI 11.42-14.18; $p=0.084 ;$ Fig.1B). Interesting, a decreased levels of plasma fibrinogen was observed in patients with IDH-1 ${ }^{\mathrm{R} 132 \mathrm{H}}$ mutation $(\mathrm{p}=0.033$, Fig.1C) and ATRX loss $(p=0.040$, Fig.1D), which a close relationship between plasma fibrinogen and malignant phenotype in GBMs.

\section{Survival analysis of plasma fibrinogen in GBMs}

The optimal cutoff of fibrinogen was calculated $3.37 \mathrm{~g} / \mathrm{L}$ for predicting OS in GBMs. The median OS of 13.83 months (95\% CI 11.70-15.96) in patients with fibrinogen $<3.37 \mathrm{~g} / \mathrm{L}$ was significantly improved than the mOS of 9.03 months (95\% CI 4.77-13.29) observed 

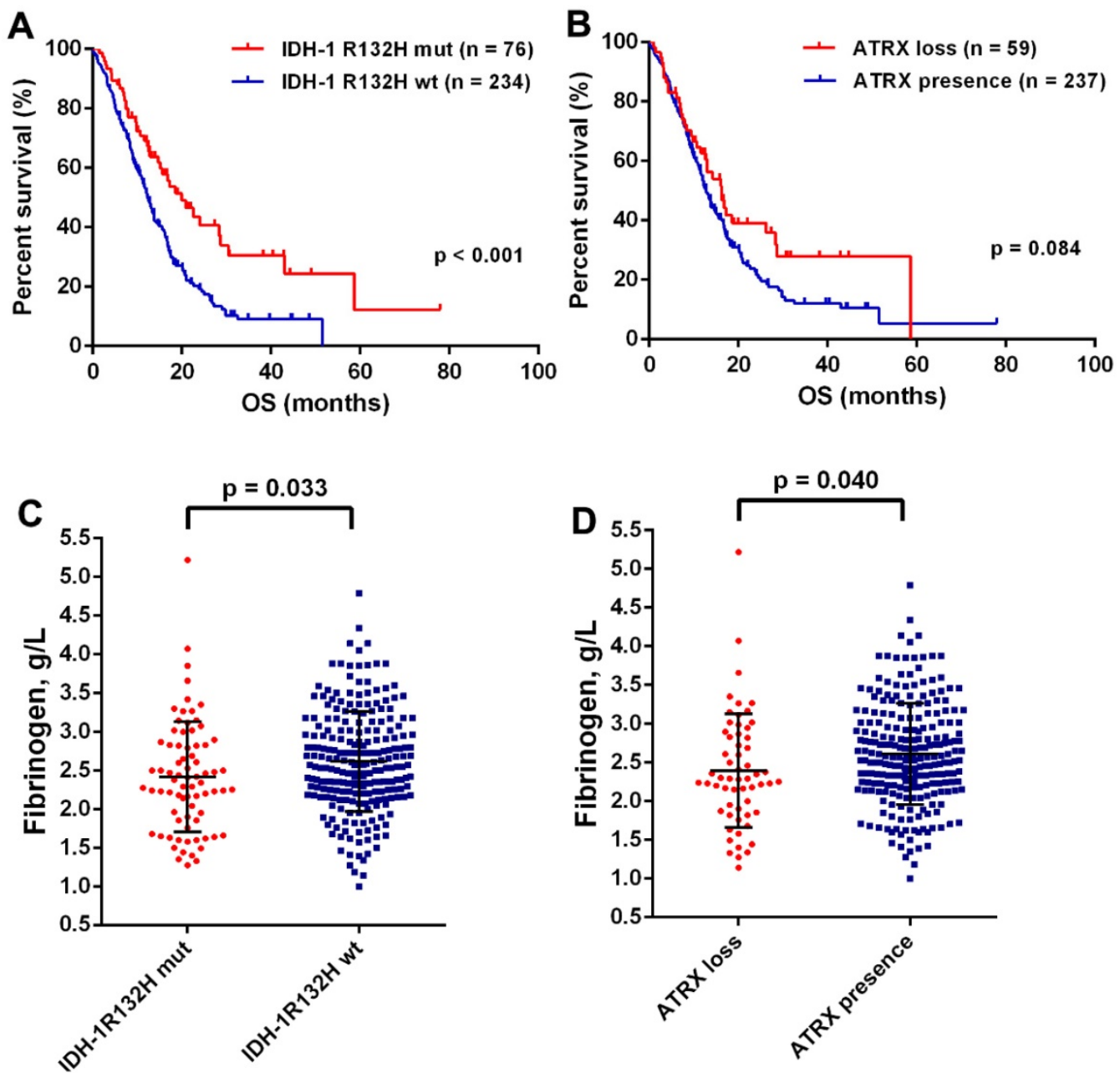

Fig. 1. (A) Kaplan-Meier curves with log rank test for patients with GBMs according to IDH mutations (A) and ATRX loss (B). The plasma fibrinogen levels were significantly increased in the group of IDH- 1 RI32H wt (C) and ATRX presence (D).

in patients of fibrinogen $\geq 3.37 \mathrm{~g} / \mathrm{L}(\mathrm{p}=0.002$, Fig.2A). IDH mutations significantly distinguished gliomas in genetics changes and survival, and served as a biomarker for GBM classification [2, 14]. Therefore, we investigated the role of plasma fibrinogen with inclusion of IDH status. In the group of IDH-1 ${ }^{\mathrm{R} 132 \mathrm{H}}$ wildtype, lower plasma fibrinogen predicted a favorable outcome [median 13.00 months $(95 \%$ CI 11.65 - 14.35) vs. 8.37 months (95\% CI 6.66 - 10.08); $\mathrm{p}=$ 0.013; Fig.2B].

The univariate analysis suggested that younger age, higher preoperative KPS, GTR, complete chemoradiotherapy, IDH-1 ${ }^{\mathrm{R} 132 \mathrm{H}}$ mutations and lower levels of fibrinogen were associated with improved OS. In the multiple analysis, we found that chemoradiotherapy, IDH-1 ${ }^{\mathrm{R} 132 \mathrm{H}}$ and fibrinogen were independent prognostic markers (Table 2).

\section{Discussion}

In the present study, we first observed that preoperative plasma fibrinogen was significantly decreased in younger patients, IDH-1 ${ }^{\mathrm{R} 132 \mathrm{H}}$ mutations and ATRX loss from 315 GBM patients' data. Our data also indicated a potential diagnostic value of plasma fibrinogen in predicting molecular subtype of GBMs. The plasma fibrinogens were independent prognostic biomarkers for OS in GBMs.

It was acknowledged that haemostatic system played a crucial role in cancer progression. A previous meta-analysis proved that higher plasma fibrinogen levels predicted worse survival in a variety of cancer [6]. However in GBMs, there were few data investigating the prognostic role of fibrinogen. Reynés $\mathrm{G}$ and colleges observed that plasma fibrinogen were significantly increased in patients with GBMs compared with heathy controls [7]. D-dimers belong to haemostatic system, negatively influence the outcome of GBMs [15]. These results supported our data that fibrinogen predicted worse prognosis in GBM. Furthermore, IDH wildtype and ATRX expression were critical markers that defined a more malignant subtype of GBMs [13]. A significant increase of plasma fibrinogen was observed in the subtype of IDH wildtype and ATRX expression, 
which also indicated a negative role of fibrinogen in GBMs.

Table 1. Association of plasma fibrinogen and clinicopathological factors

\begin{tabular}{|c|c|c|c|}
\hline \multirow[t]{2}{*}{ Variables } & \multirow[t]{2}{*}{ No. } & \multicolumn{2}{|c|}{ Plasma Fibrinogen $(\mathrm{g} / \mathrm{L})$} \\
\hline & & Mean \pm SD & P-value \\
\hline \multicolumn{4}{|l|}{ Age } \\
\hline$\geq 65$ & 46 & $2.75 \pm 0.66$ & 0.042 \\
\hline$<65$ & 269 & $2.53 \pm 0.67$ & \\
\hline \multicolumn{4}{|l|}{ Gender } \\
\hline Female & 124 & $2.57 \pm 0.63$ & 0.992 \\
\hline Male & 191 & $2.57 \pm 0.69$ & \\
\hline \multicolumn{4}{|c|}{ Preoperative KPS } \\
\hline$\geq 70$ & 203 & $2.54 \pm 0.70$ & 0.294 \\
\hline$<70$ & 112 & $2.62 \pm 0.61$ & \\
\hline \multicolumn{4}{|l|}{ Pathology } \\
\hline pGBM & 257 & $2.62 \pm 0.66$ & 0.002 \\
\hline sGBM & 58 & $2.32 \pm 0.65$ & \\
\hline \multicolumn{4}{|l|}{ Size } \\
\hline$\geq 5 \mathrm{~cm}$ & 175 & $2.61 \pm 0.71$ & 0.508 \\
\hline$<5 \mathrm{~cm}$ & 107 & $2.55 \pm 0.61$ & \\
\hline \multicolumn{4}{|l|}{ Resection } \\
\hline GTR & 196 & $2.56 \pm 0.68$ & 0.839 \\
\hline non-GTR & 109 & $2.58 \pm 0.66$ & \\
\hline \multicolumn{4}{|c|}{ Chemoradiotherapy } \\
\hline Complete & 173 & $2.56 \pm 0.63$ & 0.985 \\
\hline Incomplete & 124 & $2.56 \pm 0.67$ & \\
\hline \multicolumn{4}{|c|}{ IDH-1 R132H } \\
\hline Mutant & 77 & $2.43 \pm 0.71$ & 0.033 \\
\hline Wild-type & 238 & $2.61 \pm 0.65$ & \\
\hline \multicolumn{4}{|l|}{ ATRX } \\
\hline Loss & 60 & $2.41 \pm 0.73$ & 0.040 \\
\hline Expression & 241 & $2.61 \pm 0.65$ & \\
\hline
\end{tabular}
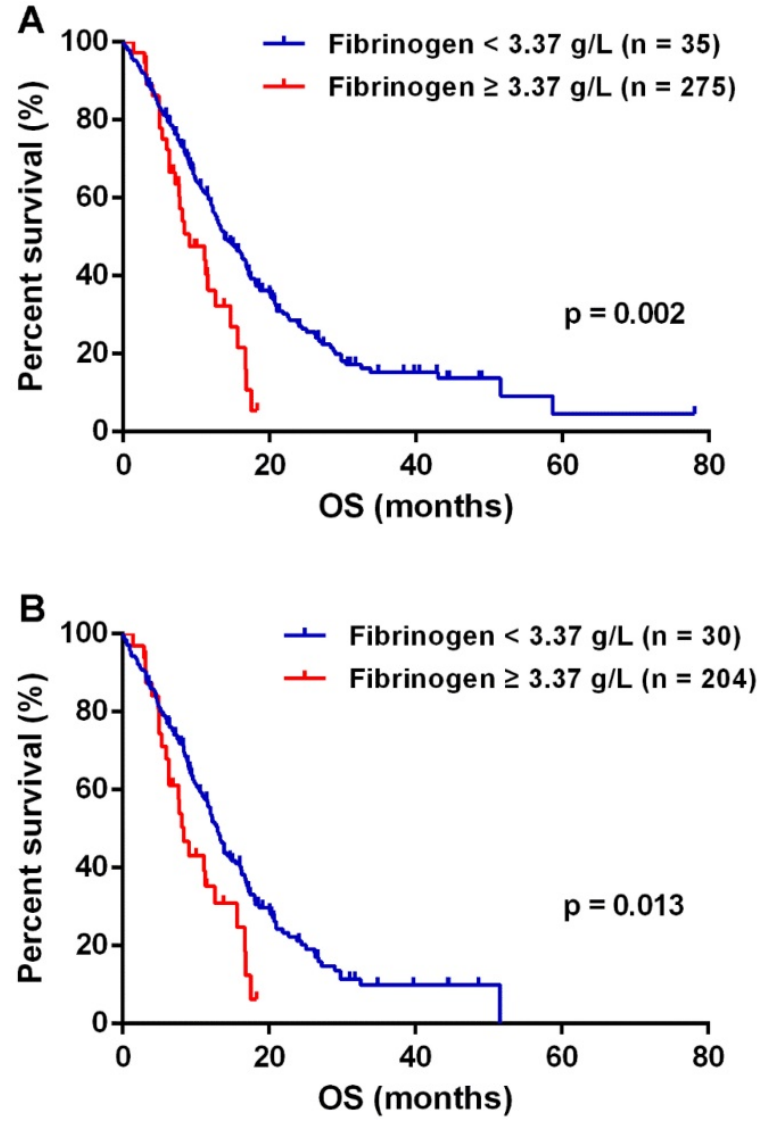

Fig. 2. Plasma fibrinogen was a prognostic factor in GBM (A) and IDHRI32H wt GBM (B) patients respectively.

Table 2. Univariate and Multivariate analysis of clinicopathological factors in relation to OS

\begin{tabular}{|c|c|c|c|c|c|c|}
\hline \multirow[t]{2}{*}{ Variables } & \multirow[t]{2}{*}{ No. } & \multicolumn{3}{|l|}{ Univariate analysis } & \multicolumn{2}{|c|}{ Multivariate analysis } \\
\hline & & $\operatorname{mOS}(95 \% \mathrm{CI})$ & HR $(95 \% \mathrm{CI})$ & $\mathrm{p}$-value & HR $(95 \% \mathrm{CI})$ & p-value \\
\hline \multicolumn{7}{|l|}{ Age } \\
\hline$\geq 65$ & 46 & $12.00(10.39-13.61)$ & $0.69(0.48-.098)$ & 0.040 & $1.02(0.69-1.51)$ & 0.922 \\
\hline$<65$ & 269 & $14.27(11.93-16.61)$ & & & & \\
\hline \multicolumn{7}{|l|}{ Gender } \\
\hline female & 124 & $12.80(9.59-16.00)$ & $1.04(0.78-1.38)$ & 0.797 & & \\
\hline male & 191 & $13.83(11.41-16.25)$ & & & & \\
\hline \multicolumn{7}{|c|}{ Preoperative KPS } \\
\hline$\geq 70$ & 203 & 16.17 (13.80-18.55) & $1.43(1.08-1.89)$ & 0.012 & $1.19(0.89-1.59)$ & 0.247 \\
\hline$<70$ & 112 & $11.56(9.72-13.41)$ & & & & \\
\hline \multicolumn{7}{|l|}{ Pathology } \\
\hline pGBM & 257 & $13.30(11.64-14.96)$ & $0.89(0.74-1.07)$ & 0.199 & & \\
\hline sGBM & 58 & $16.90(10.03-23.77)$ & & & & \\
\hline \multicolumn{7}{|l|}{ Size } \\
\hline$\geq 5 \mathrm{~cm}$ & 175 & $13.83(10.33-17.33)$ & $1.20(0.89-1.60)$ & 0.229 & & \\
\hline$<5 \mathrm{~cm}$ & 107 & $13.00(9.31-16.70)$ & & & & \\
\hline \multicolumn{7}{|l|}{ Resection } \\
\hline GTR & 196 & 14.70 (12.04-17.36) & $1.40(1.06-1.86)$ & 0.019 & $1.22(0.91-1.64)$ & 0.180 \\
\hline non-GTR & 109 & $11.33(7.63-15.04)$ & & & & \\
\hline \multicolumn{7}{|c|}{ Chemoradiotherapy } \\
\hline Complete & 173 & $18.00(15.07-20.94)$ & $3.28(2.45-4.41)$ & $<0.001$ & $3.77(2.76-5.14)$ & $<0.001$ \\
\hline Incomplete & 124 & $7.50(5.47-9.53)$ & & & & \\
\hline \multicolumn{7}{|c|}{ IDH-1 R132H } \\
\hline Mutant & 77 & $19.93(13.62-26.24)$ & $1.91(1.35-2.71)$ & $<0.001$ & $2.54(1.72-3.74)$ & $<0.001$ \\
\hline Wild-type & 238 & $12.30(10.90-13.70)$ & & & & \\
\hline \multicolumn{7}{|l|}{ ATRX } \\
\hline Loss & 60 & $16.23(12.16-20.30)$ & $1.41(0.98-2.03)$ & 0.066 & & \\
\hline Expression & 241 & $12.80(11.42-14.18)$ & & & & \\
\hline \multicolumn{7}{|l|}{ Fib } \\
\hline$\geq 3.37$ & 35 & $9.03(4.77-13.29)$ & $0.54(0.35-0.83)$ & 0.005 & $0.64(0.41-1.00)$ & 0.048 \\
\hline$<3.37$ & 280 & $13.83(11.70-15.96)$ & & & & \\
\hline
\end{tabular}


There were several mechanisms for elucidating the malignant role of fibrinogen in gliomas. The first was the crosstalk between inflammation and fibrinogen [16]. It was found that fibrinogen could activate leukocyte via integrin $\alpha_{M} \beta_{2}$ [17]. The main leukocyte population was neutrophils, which secreted arginase-1 and VEGF (vascular endothelial growth Factors) for immunosuppression and angiogenesis respectively in gliomas [18]. Meanwhile, NK cell function was negatively influenced by fibrinogen, and thus unable to kill tumor cells [16]. There was evidence suggested that tumor associated tissue factors (TFs) could lead to tumor metastasis depended on fibrinogen [19]. TFs and fibrinogen were both detected in gliomas, associated with a worse clinical outcome, and contributed to gliomagenesis [20]. Interestingly, intratumoral coagulation-related gene was differently expressed in GBMs, and was most abundant in mesenchymal subtype [21]. IDH mutations were rarely detected in mesenchymal subtype [21], and most likely to positively correlate with coagulation-related gene expression. So there might exist a cooperation between circulating and intratumoral fibrinogens, and promote glioma development. Further studies were needed to clarity this relationship.

Recent studies suggested that blood biomarkers such as NLR [5], albumin [22] nutritional index [23] or their combinations, could also predict clinical outcome in patients with GBMs. These circulating biomarkers had far-reaching significance as they were cost-effective, mini-invasive and clinically informative. Moreover, the combination of NLR and LMR could provide diagnosis value of distinguishing gliomas from other brain tumours (acoustic neuroma and meningiomas) [24]. Additionally, we also showed a strong correlation of fibrinogen with key genetic alternations in GBMs. Our results were supported by the acknowledged concepts that tumor cells could evolve due to the active interactions between tumor cells and blood hemostatic factors [16]. However, most studies above were retrospective and single centres. The cut-off of the blood biomarkers hardly unified either, which limited the clinical practice. So prospective multiple-centres studies, with rational cut-off were needed.

In conclusion, our study first proved an independent prognostic role of fibrinogen in GBMs. The higher plasma fibrinogen levels could also predict the co-occurrence of IDH wildtype and ATRX expression in GBMs. We suggested that physicians could take use of fibrinogen in everyday clinical practice, as it was easily detected in every patient.

\section{Acknowledgement}

This work was supported by grants from National Key Technology Research and Development Program of the Ministry of Science and Technology of China (No.2014BAI04B01) and the Natural Science Foundation of Beijing (No. 7182076).

\section{Competing Interests}

The authors have declared that no competing interest exists.

\section{References}

1. Ostrom QT, Gittleman H, Xu J et al. CBTRUS Statistical Report: Primary Brain and Other Central Nervous System Tumors Diagnosed in the United States in 2009-2013. Neuro Oncol. 2016; 18:v1-v75.

2. Louis DN, Perry A, Reifenberger G et al. The 2016 World Health Organization Classification of Tumors of the Central Nervous System: a summary. Acta Neuropathol. 2016; 131:803-820.

3. Woehrer A, Bauchet L, Barnholtz-Sloan JS. Glioblastoma survival: has it improved? Evidence from population-based studies. Curr Opin Neurol. 2014; 27:666-674.

4. Cai J, Zhu P, Zhang $\mathrm{C}$ et al: Detection of ATRX and IDH1-R132H immunohistochemistry in the progression of 211 paired gliomas. Oncotarget. 2016; 7(13):16384-95

5. Mei Z, Shi L, Wang B et al. Prognostic role of pretreatment blood neutrophil-to-lymphocyte ratio in advanced cancer survivors: A systematic review and meta-analysis of 66 cohort studies. Cancer Treat Rev. 2017; 58:1-13.

6. Perisanidis C, Psyrri A, Cohen EE et al. Prognostic role of pretreatment plasma fibrinogen in patients with solid tumors: A systematic review and meta-analysis. Cancer Treat Rev. 2015; 41:960-970.

7. Reynes G, Vila V, Martin M et al. Circulating markers of angiogenesis, inflammation, and coagulation in patients with glioblastoma. J Neurooncol. 2011; 102:35-41.

8. He ZQ, Duan H, Ke C et al. Evaluation of cumulative prognostic score based on pretreatment plasma fibrinogen and serum albumin levels in patients with newly diagnosed high-grade gliomas. Oncotarget. 2017; 8: 49605-49614.

9. Stupp R, Mason WP, van den Bent MJ et al: Radiotherapy plus concomitant and adjuvant temozolomide for glioblastoma. N Engl J Med. 2005; 352:987-996.

10. Bambury RM, Teo MY, Power DG et al. The association of pre-treatment neutrophil to lymphocyte ratio with overall survival in patients with glioblastoma multiforme. J Neurooncol 2013; 114:149-154.

11. Wang PF, Chen Y, Song SY et al. Immune-Related Adverse Events Associated with Anti-PD-1/PD-L1 Treatment for Malignancies: A Meta-Analysis. Front Pharmacol. 2017; 8:730.

12. Budczies J, Klauschen F, Sinn BV et al. Cutoff Finder: a comprehensive and straightforward Web application enabling rapid biomarker cutoff optimization. PLoS One. 2012; 7:e51862.

13. Pekmezci M, Rice T, Molinaro AM et al: Adult infiltrating gliomas with WHO 2016 integrated diagnosis: additional prognostic roles of ATRX and TERT. Acta Neuropathol. 2017; 133:1001-1016.

14. Yan H, Parsons DW, Jin G et al: IDH1 and IDH2 mutations in gliomas. N Engl J Med. 2009; 360:765-773.

15. Hoke M, Dieckmann K, Koppensteiner R et al. Prognostic value of plasma d-dimer levels in patients with glioblastoma multiforme - Results from a pilot study. Wien Klin Wochenschr. 2011; 123:199-203.

16. Degen JL, Palumbo JS. Hemostatic factors, innate immunity and malignancy. Thromb Res. 2012; 129 Suppl 1:S1-5.

17. Steinbrecher KA, Horowitz NA, Blevins EA et al. Colitis-associated cancer is dependent on the interplay between the hemostatic and inflammatory systems and supported by integrin alpha(M)beta(2) engagement of fibrinogen. Cancer Res. 2010; 70:2634-2643.

18. Massara M, Persico P, Bonavita O et al. Neutrophils in Gliomas. Front Immunol. 2017; 8:1349.

19. Palumbo JS, Talmage KE, Massari JV et al. Tumor cell-associated tissue factor and circulating hemostatic factors cooperate to increase metastatic potential through natural killer cell-dependent and-independent mechanisms. Blood. 2007; 110:133-141.

20. Magnus N, Meehan B, Garnier D et al. The contribution of tumor and host tissue factor expression to oncogene-driven gliomagenesis. Biochem Biophys Res Commun. 2014; 454:262-268.

21. Verhaak RG, Hoadley KA, Purdom E et al: Integrated genomic analysis identifies clinically relevant subtypes of glioblastoma characterized by abnormalities in PDGFRA, IDH1, EGFR, and NF1. Cancer Cell. 2010; 17:98-110.

22. Han S, Huang $\mathrm{Y}, \mathrm{Li} \mathrm{Z}$ et al. The prognostic role of preoperative serum albumin levels in glioblastoma patients. BMC Cancer. 2015; 15:108 
23. Zhou XW, Dong $\mathrm{H}$, Yang $\mathrm{Y}$ et al. Significance of the prognostic nutritional index in patients with glioblastoma: A retrospective study. Clin Neurol Neurosurg. 2016; 151:86-91.

24. Huang J, Yuan Y, Wang Y et al. Preoperative prognostic nutritional index is a significant predictor of survival in patients with localized upper tract urothelial carcinoma after radical nephroureterectomy. Urol Oncol. 2017; 35: 671.e1-671.e9. 